\title{
Complete genome sequencing and network modeling to overcome trastuzumab resistance
}

\begin{abstract}
"What are the challenges for completing the driver mutations catalog and how can we predict the complex nonlinear relationship between these driver mutations and the phenotype of individual patients towards the development of a next generation of biomarkers and biologic agents?"
\end{abstract}

\section{KEYWORDS: breast $\approx$ cancer genome $\approx$ gastric cancer $\approx$ molecular networks - trastuzumab resistance}

Despite advances in our understanding of the molecular basis of traditional single genes and the clinical success of trastuzumab for treating HER 2 overexpressing breast and gastric tumors, resistance to trastuzumab plus chemotherapy treatment failure in HER2-negative tumors and death rates are still alarmingly high. These limitations are now explained by the latest data from sequencing technologies [1-5], which reveal a higher complexity and heterogeneity of breast cancer than we originally thought [6]. What are the challenges for completing the driver mutations catalog and how can we predict the complex nonlinear relationship between these driver mutations and the phenotype of individual patients towards the development of a next generation of biomarkers and biologic agents?

\section{Cancer development \& metastasis}

With nearly 2 million new diagnoses and over 1.2 million deaths each year worldwide [7], breast cancer and gastric cancer are major health problems. In the USA, breast cancer is, and will probably continue to be, the most common malignancy among females, while gastric cancer incidence has been dramatically reduced in the USA, but its global incidence and mortality still remain alarmingly high [8].

Cancer initiation, progress and metastasis are driven by mutations in genes that are key components in the signaling pathways. These causal so-called 'driver' mutations deregulate signal transduction from outside the cell through cell receptors to the nucleus. This deregulation of cell proliferation, growth, cell death (apoptosis) and angiogenesis results in cancer development and metastasis. By inhibiting key signaling pathways responsible for cancer progression through drugs, cancer progression in the metastatic setting and recurrence risk in the adjuvant setting could successfully be treated. This is a characteristic example of translating this targeted therapy concept to breast and gastric cancer treatment with the biologic agent, trastuzumab.

“... a previous randomized controlled trial (RCT) for breast cancer and the recent international Phase III RCT for gastric cancer, have provided evidence for the safety and efficacy of trastuzumab added to chemotherapy to significantly prolong overall survival..."

\section{Trastuzumab}

The Erbb family plays a crucial part in the development and evolution of cancer. This receptor family consists of four closely related type 1 transmembrane tyrosine kinase receptors: EGF receptor (EGFR; also known as HER1), ERBB2 (HER2), ERBB3 (HER3) and ERBB4 (HER4). Each receptor comprises an extracellular domain at which ligand binding occurs, an $\alpha$-helical transmembrane segment and an intracellular protein tyrosine kinase domain. Ligand binding to these EGF family of receptors phosphorylates and activates a complex intracellular signaling pathway network that controls a range of cellular processes, including proliferation, angiogenesis, cell cycle, survival and apoptosis [9].

HER2 amplification and overexpression plays a central role in the initiation, progression and metastasis of some common cancers, including breast and gastric cancer [10,11]. HER2 status has been recognized as an important prognostic factor. Patients with breast or gastric cancer and HER2-positive tumors have a significantly lower

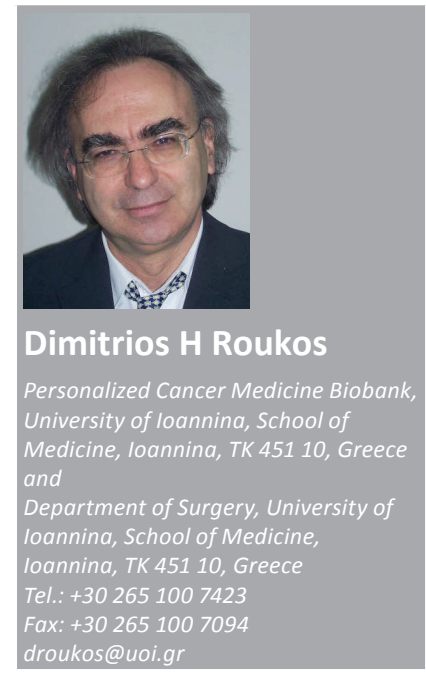

future medictio $^{\text {pisg }}$ fsg 
chance of survival than those with HER2-negative tumors [9-11]. Thus, this pivotal receptor could be a potential therapeutic target.

Trastuzumab is a monoclonal antibody that on binding to the extracellular domain of HER2, inhibits the HER2 signaling pathway activity in tumor cells overexpressing HER2. In the metastatic setting, a previous randomized controlled trial (RCT) for breast cancer [10] and the recent international Phase III RCT for gastric cancer [11], have provided evidence for the safety and efficacy of trastuzumab added to chemotherapy to significantly prolong overall survival in patients with these two major cancer types and HER2-positive tumors. For advanced, metastatic or recurrent HER2-positive gastric cancer, trastuzumab-based systemic treatment with chemotherapy has become a new standard of care [11-13].

In the adjuvant setting, trastuzumab for early stage breast cancer represents the triumph of bench-to-bedside molecular research [14]. Indeed, potential clinical success at this setting can save the lives of thousands of patients with nonmetastatic disease. Five RCTs with HER2-positive early stage breast cancer comparing adjuvant systemic chemotherapy with and without trastuzumab in 13,493 patients have been reported. An updated meta-analysis has shown a significant reduction in risk recurrence by $38 \%$ and death by $34 \%$ [15]. Although the efficacy of trastuzumab in improving rates of both disease-free survival and overall survival now appears clear, results from longer follow-up are needed in order to be able to draw definitive conclusions [16].

\section{Limitations \& explanation}

Currently, intrinsic and acquired resistance to anti-tumor treatment is the biggest challenge. No response to trastuzumab has been reported in HER2-negative patients, who account for approximately $75 \%$ of all breast or gastric cancers. The net response rate among HER2-positive patients with breast or gastric cancer is less than $15 \%$, plus the approximately $30 \%$ response rate to chemotherapy suggests that approximately $45 \%$ of patients with HER2-overexpressing tumors benefit from trastuzumab-chemotherapy treatment [10-15]. Administration of trastuzumab was associated with increased risks for congestive heart failure, left ventricular ejection fraction decline and CNS metastasis as the first recurrence event in breast cancer [15]. In addition, given the short-term follow-up, it is unclear whether trastuzumab may have a long-term efficacy leading to cure or if it has only a delaying effect on reoccurence.

How can we explain the limited effectiveness of trastuzumab? There are two main causes of current failure in the targeted treatment of breast and gastric cancer. First, the lack of robust biomarkers for predicting response to specific therapies. Second, the discovery of key targets for the development of effective drugs has not yet been achieved. For example, in HER2-negative tumors, the need to identify which signaling pathways have been activated is clear and research is underway either to predict which patients may respond to anti-VEGF agents or to identify other signaling pathways, beyond the EGFR and VEGF pathways, which are activated in HER2-negative tumors. Among HER2-positive patients, it appears that in a subset of patients the HER2 pathway plays the predominant role in disease progression, while in the remaining patients the oncological outcome is driven by either other pathways than HER2 downstream pathways or by interactions (interconnections) of several signaling pathways. Based on emerging evidence from the latest large-scale studies using next-generation DNA sequencing technology, which reveals that high heterogeneity and complexity of breast and gastric cancer exist $[6,17,18]$, new research directions are being shaped to overcome resistance to trastuzumab.

“...systematic studies of more than 25,000 cancer genomes at the genomic, epigenomic and transcriptomic levels will reveal the repertoire of oncogenic mutations ... and enable the development of new cancer therapies."

\section{'Big' biology}

From the first draft of a complete human genome sequence by the Human Genome Project a decade ago to the recently launched International Cancer Genome Consortium (ICGC) [19], our understanding of tumor pathogenesis and progression has dramatically improved. Although clinical implications are still limited [1-5], latest advances in sequencing technology allow us to carry out more systematic and rational studies towards gaining an in-depth understanding of the genetic basis of cancer. Comparing the genome sequence of tumor cells with that of normal cells can reveal genetic differences between normal cells 
and cancer cells. These DNA changes, including point mutations, genomic rearrangements and copy number changes, can now be faster, cheaper and reliably assessed by current sequencing technology. However, the more we learn, the more questions than answers will emerge $[4,5]$. The repertoire of oncogenic mutations for each cancer type is very large, and because of wide genetic variability, patients with the same phenotype, namely clinical/pathological features, have different prognoses and various responses to therapies. Moreover, another international collaborative project called the Encyclopedia of DNA Elements (ENCODE) showed that in a selected portion of the genome containing just a few percent of protein-coding sequence, between 74 and $93 \%$ of DNA was transcribed into RNA. Much noncoding DNA plays a regulatory role; small RNAs of different varieties seem to control gene expression at the level of both DNA and RNA transcripts in ways that are only now beginning to become clear $[4,5]$.

\section{Complete cancer genome sequencing}

The vast majority of mutations fall into the noncoding DNA - what used to be called 'junk' DNA [5]. The full genome sequence in a woman with breast cancer showed 27,173 point mutations in noncoding regions versus 200 in coding DNA from the primary tumor, and 51,710 versus 225 point mutations in brain metastases, respectively [6]. However, no one knows the function of the nonprotein-coding genome and whether these mutations are being causatively involved in cancer. Given the high costs and effort included, there is currently a debate regarding whether it is better to systematically investigate the fullgenome sequence now, or later when the costs will be substantially lower [5].

According to scientists of the ICGC [19], systematic studies of more than 25,000 cancer genomes at the genomic, epigenomic and transcriptomic levels will reveal the repertoire of oncogenic mutations, uncover traces of the mutagenic influences, define clinically relevant subtypes for prognosis and therapeutic management, and enable the development of new cancer therapies.

In addition, large-scale projects are being run in parallel with the cancer-sequencing consortia to assess the effects of deleting each gene in the mouse genome, enabling researchers to learn more about the normal function of genes that are mutated in cancer. Moreover, other projects aiming to improve our understanding of signaling pathway interactions within a single cancer cell, as well as between cancer cells and stromal cells in an individual tumor are being carried out. All these cancer-sequencing projects and systems biology approaches will provide important insights into biological processes and molecular mechanisms that drive tumor initiation and metastasis, but to what extent will they change oncology practice?

\section{Linking phenotype to genotype}

Both complete genotype and phenotype data are crucial in the effort to predict the risk of recurrence or disease progression and survival. Several studies have already identified a large number of mutations and genes involved in cancer development and metastasis. With cheaper and refined sequencing technology, the number of whole and partial cancer genomes sequenced will dramatically be increased, improving the catalog of driver mutations, including point mutations, genomic rearrangements and copy number changes $[6,17,18]$. From a phenotype perspective regarding individual patient's profile, high-quality clinical, pathological, therapeutic and followup data (phenotype) are available from large-scale RCTs and databases [20,21]. Although still in its infancy, effort is underway to discover how to link a phenotypic event (e.g., recurrence or death) with genetic alterations (e.g., genotype) [3,22-26].

\section{"...it is important not only to characterize which pathways are activated in which patient, but also what is the ultimate outcome of the signaling pathways networks."}

Yet the understanding of a nonlinear complex relationship between genotype and phenotype is a major problem. Rapid progress and collaboration between researchers in biomedical and mathematical sciences may overcome current challenges. Several computational strategies are being developed to predict gene-gene and gene-environment interactions [20-24]. Bionetworks modeling represents one of the most fascinating fields towards a genotype-phenotype-based personalized medicine [27]. Efforts are underway to integrate genotyping and molecular data into molecular networks modeling to predict outcomes [22,23]. Systems biology approaches shape a new method to understand complex biological systems [28], such as individual tumor, host and environment [29]. The goal is to link genomic data with clinical data to understand why some patients respond to therapy and are cured, whereas others 
do not respond to therapy and experience fatal metastatic recurrence. Given that the mutations catalog has to be completed, bionetworks modeling with an emphasis on completed clinical data available might more quickly lead to the development of the next generation of networkbased biomarkers and biologically targeted agents [24,29].

Beyond the ERBB family and VEGF, other signalling pathways, for example, the Wnt, Notch, Hedgehog and TGF pathways may also have a cruical role in cancer and the activation of these pathways may vary among patients with the same tumor and stage [30,31]. Indeed, a recent study using new sequencing technology has revealed that in pancreatic cancer approximately 12 signaling pathways are activated for each patient, and there is a widespread variability of deregulated downstream pathways [31]. Given the interconnections of the signaling pathways components [30], and the deregulated pathways heterogeneity, it is important not only to characterize which pathways are activated in which patient, but also what is the ultimate outcome of the signaling pathways networks. Therefore, a systems biology approach to understanding signaling pathway networks for the prediction of signal transduction, which includes input (receptors)-intracellular signaling pathways network output (nucleus), is crucial for a new generation of biomarkers and biologic agents [32].

\section{Bibliography}

Papers of special note have been highlighted as:

- of interest

-11 of considerable interest

1 The human genome at ten. Nature 464(7289), 649-650 (2010).

2 Collins F: Has the revolution arrived? Nature 464(7289), 674-675 (2010).

- Genome sequencing perspectives to improve health by pioneering new methods in public human genome sequencing.

3 Venter JC: Multiple personal genomes await. Nature 464(7289), 676-677 (2010).

- Genome sequencing perspectives to improve health by pioneering new methods in public human genome sequencing.

4 Check Hayden E: Human genome at ten: life is complicated. Nature 464(7289), 664-667 (2010)

5 Ledford H: Big science: the cancer genome challenge. Nature 464(7291), 972-974 (2010).
6 Ding L, Ellis MJ, Li S et al.: Genome remodelling in a basal-like breast cancer metastasis and xenograft. Nature 464(7291), 999-1005 (2010).

- $\quad$ First report on complete genome sequencing and data comparison of the primary and metastatic tumors in a woman with breast cancer.

7 Kamangar F, Dores GM, Anderson WF: Patterns of cancer incidence, mortality, and prevalence across five continents: defining priorities to reduce cancer disparities in different geographic regions of the world. J. Clin. Oncol. 24(14), 2137-2150 (2006).

8 Jemal A, Siegel R, Ward E et al.: Cancer statistics 2009. CA Cancer J. Clin. 59, 225-249 (2009).

9 Baselga J, Swain SM: Novel anticancer targets: revisiting ERBB2 and discovering ERBB3. Nat. Rev. Cancer 9(7), 463-475 (2009).

10 Slamon DJ, Leyland-Jones B, Shak S et al.: Use of chemotherapy plus a monoclonal antibody against HER2 for metastatic breast cancer that overexpresses HER2. N. Engl. J. Med. 344, 783-792 (2001).

\section{Conclusion}

Trastuzumab in conjunction with chemotherapy provides an overall survival benefit in advanced and metastatic breast and gastric cancer, and reduces the risk of recurrence in the breast cancer adjuvant setting. However, mortality rates for these diseases remain alarmingly high.

At the end of the first post-genomic decade, with an explosion in latest genome-sequencing technology, current evidence reveals that the complexity and heterogeneity of breast and gastric cancer. Now, two innovate strategies are being developed: the completion of the breast and gastric cancer driver mutations catalog, and the understanding of the complex genotypephenotype map through network modeling. Although these research directions provide a scientifically rational promise for the next generation of robust biomarkers and effective biologic drugs, many challenges have to be overcome in order to achieve them.

\section{Financial \& competing interests disclosure}

The author has no relevant affiliations or financial involvement with any organization or entity with a financial interest in or financial conflict with the subject matter or materials discussed in the manuscript. This includes employment, consultancies, honoraria, stock ownership or options, expert testimony, grants or patents received or pending, or royalties.

No writing assistance was utilized in the production of this manuscript.

11 van Cutsem E, Kang Y, Chung $\mathrm{H}$ et al.: Efficacy results from the ToGA trial: a Phase III study of trastuzumab added to standard chemotherapy (CT) in first-line human epidermal growth factor receptor 2 (HER2)-positive advanced gastric cancer (GC). J. Clin. Oncol. 27(Suppl.), 18S (2009) (Abstract No. LBA4509).

" First large-scale randomized trial on trastuzumab in advanced gastric cancer.

12 Roukos DH: Targeting gastric cancer with trastuzumab: new clinical practice and innovative developments to overcome resistance. Ann. Surg. Oncol. 17, 14-17 (2010).

13 Petrelli NJ, Winer EP, Brahmer J et al. Clinical cancer advances 2009: major research advances in cancer treatment, prevention, and screening - a report from the American Society of Clinical Oncology. J. Clin. Oncol. 27(35), 6052-6069 (2009).

14 Hortobagyi GN: Trastuzumab in the treatment of breast cancer. N. Engl. J. Med. 353(16), 1734-1736 (2005). 
15 Dahabreh IJ, Linardou H, Siannis F, Fountzilas G, Murray S: Trastuzumab in the adjuvant treatment of early-stage breast cancer: a systematic review and meta-analysis of randomized controlled trials. Oncologist 13(6), 620-630 (2008).

16 Roukos DH: Personalized cancer diagnostics and therapeutics. Expert Rev. Mol. Diagn. 9(3), 227-229 (2009).

17 Stephens PJ, McBride DJ, Lin ML et al.: Complex landscapes of somatic rearrangement in human breast cancer genomes. Nature 462 (7276), 1005-1010 (2009).

- New method revealing the important role of somatic rearrangement in breast cancer.

18 Beroukhim R, Mermel CH, Porter D et al: The landscape of somatic copy-number alteration across human cancers. Nature 463(7283), 899-905 (2010).

- Landmark study on the crucial role copy number changes across human cancers.

19 International Cancer Genome Consortium: International network of cancer genome projects. Nature 464, 993-998 (2010).

20 Ziogas D, Roukos DH: Genetics and personal genomics for personalized breast cancer surgery: progress and challenges in research and clinical practice. Ann. Surg. Oncol. 16(7), 1771-1782 (2009).
21 Roukos DH: Genome-wide association studies and aggressive surgery toward individualized prevention, and improved local control and overall survival for gastric cancer. Ann. Surg. Oncol. 16(4), 795-798 (2009).

22 Rockman MV: Reverse engineering the genotype-phenotype map with natural genetic variation. Nature 456, 738-744 (2008).

23 Bohman T: Mathematics. Emergence of connectivity in networks. Science 323(5920), 1438-1439 (2009).

24 Roukos DH: Novel clinico-genome network modeling for revolutionizing genotype-phenotype-based personalized cancer care. Expert Rev. Mol. Diagn. 10 (1), 33-48 (2010).

- Novel model with emphasis on clinical data in approaching genotype-phenotype map towards personalized cancer medicine.

25 Schadt EE: Molecular networks as sensors and drivers of common human diseases. Nature 461(7261), 218-223 (2009).

26 Roukos DH: Systems medicine: a real approach for future personalized oncology? Pharmacogenomics 11(3), 283-287 (2010).

27 Roukos DH: Bionetworks-based personalized medicine versus comparative-effectiveness research or harmonization of both in cancer management? Expert Rev. Mol. Diagn. 10(3), 247-250 (2010).
28 Weinberg R: Point: hypotheses first. Nature 464(7289), 678 (2010).

29 Roukos DH, Katsios C, Liakakos T: Genotype-phenotype map and molecular networks: a promising solution in overcoming colorectal cancer resistance to targeted treatment. Expert Rev. Mol. Diagn. 10(5), 541-545 (2010).

30 Hahn WC, Weinberg RA: Modelling the molecular circuitry of cancer. Nat. Rev. Cancer 2(5), 331-341 (2002).

31 Jones S, Zhang X, Parsons DW: Core signaling pathways in human pancreatic cancers revealed by global genomic analyses. Science 321(5897), 1801-1806 (2008).

32 Roukos DH: Beyond HER2 and trastuzumab: heterogeneity, systems biology, and cancer origin research may guide the future for personalized treatment of very early but aggressive breast cancer. J. Clin. Oncol. 28(17), E279-E280 (2010). 